\section{SUMMER DIARRHEA OF INFANTS.}

ITS ETIOLOGY AND TREATMENT.

BY H. M. MCCLANAHAN, A.M., M.D.

PROFESSOR OF DISEASES OF CHILDREN, OMAHA MEDICAL COLLEGE. OMAHA, NEB.

While the summer diarrheas of infants vary much in their clinical manifestation and in the pathologic lesions, they are all similar in their etiology and treatment. It is, therefore, to these phases of the subject that I wish to direct attention.

\section{ETIOLOGY.}

The etiologic factors are predisposing and exciting, the former are age, environment, season, character and method of feeding, and previous condition of health. Nearly all cases occur during the first two years of life. During this period the child has feeble resisting power; its digestive organs are readily prone to functional disturbances. Its nervous system has feeble inhibitory power, and the irritation that in the adult would have no effect, in the infant produces profound impressions.

The influence of environment is particularly noted in large eities where, owing to the lack of fresh air and sunshine there is induced a feebleness of constitution that increases the susceptibility of the child to morbific influences.

The method of feeding and the quality of the food is a nearly constant predisposing cause. Statistics show that about 97 per cent. of all cases occur in bottle-fed babies.

The predisposing element is undoubtedly the quality of the food, in most cases lacking in elements to symmetrieally nourish the child, and, by producing an enfeebled condition, make it more vulnerable to the exciting cause. Rickets is a conspicuous example.

Again, the food may be difficult of digestion, as when it contains an excess of albuminoids. In these cases, the digestive organs are taxed beyond their capacity and a state of chronic irritation produced. It can be safely affirmed that the majority of cases of summer diarrhea are preceded by some form of indigestion due to the method of feeding. The season is so important that these diseases were formerly looked upon as thermic ferers. We now know that they are not directly induced by hrat.

Modern bacteriology has taught us the true influence of heat in inducing these disease. We should not, however, lose sight of the fact that extreme heat is debilitating, that its enervating influence lessens the resisting power, that the profuse perspiration creates a thirst which leads the child to ingest excessive quantities of food, overloading the digestive organs, and impairing their functional activity. 'The previous condition of the health of a child is perhaps more important in influencing our prognosis than in causing summer diarrhea.

The child whose constitution is enfeebled by sickness, either acute or chronic, is not only more susceptible to these diseases, but is less able to withstand the invasion. All the conditions I have mentioned exert, either singly or together, an influence in predisposing the child to summer diarrhea but none or all together, in the true sense of the term, cause these conditions.

Summer diarrhea is an infectious disease, just as certainly and upon the same evidence as typhoid fever, with the distinction, however, that it is not due to one specific germ. The method of invasion is nearly always by the food through the gastro-intestinal tract. The poison originates in the milk, through the agency of bacterial growth.

I quote from Professor Vaughan of Ann Arbor ${ }^{1}$ :
"The child taking its nourishment directly from the breast of the healthy mother obtains its milk practically germ-free, while the one taking cow's milk receives along with this food many kinds of bacteria, some of which are very harmful. These diseases are confined to the summer months because the germs which elaborate poisons in milk require a relatively high temperature for their growth. During the hot months of summer these bacteria are widely distributed, and easily find their way into milk. They grow rapidly and produce chemical poisons. Furthermore, decomposing matter harbors and supports these bacteria at a time when the outdoor temperature is high enough to allow their growth."

The intestinal tract of healthy children contains bacteria. The two varieties constantly found are bacillus lactis aerogenes, and bacillus coli communis. As to the part played by these bacteria in normal digestion, but little is positively known.

In all forms of diarrhea these obligatory milk-feces bacteria are greatly increased in number, and are more widely distributed throughout the alimentary tract.

According to Booker's investigation, in mild types of summer diarrhea these varieties predominate almost to the exelusion of all others. Owing to their increased number they develop acids in the intestinal tractlactic, acetic and butyric. These acids irritate the mucous membrane and are probably the direct cause of the diarrhea. More important, however, is the fact that by reason of the irritation of the mucous membrane from the increased number of these bacteria, it is made more vulnerable to the influence of virulent pathogenic germs. In the more serious types of diarrhea, however, other bacteria are present, among the most common being the proteus vulgaris.

In many of these serious cases, as post-mortem examinations demonstrate, the actual damage is the result of bacterial growth, since no lesion of the intestinal wall is discovered and no penetration of the body tissues by bacteria can be demonstrated. In this class of cases the symptoms are certainly to be attributed to the absorption of toxins. In cases where the micrococci are present there are usually found distinct inflammatory changes in the intestinal mucous membrane. It is most generally the staphylococci that are found. These bacteria may find an entrance into the subjacent tissues, and thence may be distributed throughout the body. When these cases do not terminate fatally in the acute stage, they are likely to drag along for weeks and even months. "It is the consensus of opinion among investigators that no one specific kind but many different kinds of bacteria are concerned in the causation of summer diarrhea." ${ }^{2}$ Indeed, as many as forty different varieties have been detected.

A further important fact is this: chemical poisons, the result of bacterial growth, may be developed in the milk before it is taken into the stomach and these poisons may induce very serious symptoms in a short time after the milk is ingested. Professor Vaughan reports a case where symptoms of cholera infantum developed within two hours after taking the milk.

There is certainly good reason for believing that true cholera infantum is caused by toxins developed outside the body. It is important to remember that the stomach of the infant has feebler digesting power than that of the adult; that the albuminoids of milk are but slightly changed in the stomach, but pass into the bowel almost

\footnotetext{
I Edwards' Supplement to Keating's Cyclopedia, p. 15.

2 Karting's Cyclopedia, Vol. iii, p. 190. Intestinal Bacteria in Children-Booker.
} 
unaltered, hence, poisons in the milk are not likely to be destroyed or inhibited by the gastric ferments.

\section{TREATMENT.}

'The first and most important principle of treatment is to stop milk and all other kinds of food. This direction should be made mandatory, because people, in mistaken kindness, will insist upon giving the child some nourishment. The reasons for this are that milk affords a pabulum for development and growth of bacteria, and therefore tends directly to increase and continue the difficulty. Furthermore, digestion is greatly impaired and the child receives little nourishment from the food. The next object is to unload the intestinal tract as completely as possible. This is done to remove the supply of poison, lessen the absorption of toxins, and prevent irritation of the mucous membrane by the contact of decomposing material. The means to this end are mechanical and medicinal.

The mechanical means are lavage of the stomach and irrigation of the bowels. In those cases where there is marked gastric irritation, lavage is an excellent mode of treatment, not only because it empties the stomach, but for the reason that it prevents vomiting. While some writers say this is entirely free from danger, yet if a serious result had ever occurred such a statement is incorrect. One fatal case has been reported from introducing the catheter into the larynx. To avoid this accident, the index finger should be used as a tongue depressor, when the eatheter can be carried over the base of the tongue, and against the posterior wall of the pharynx, and thence on down the esophagus. In this way all danger can be avoided.

Irrigation of the bowels reaches directly only the large intestine, and in order to accomplish this purpose, it is necessary that large quantities of water be used, at least from half a gallon to a gallon. As the object is to flush out the colon the operation is to be continued until the water returns free from fecal matter. While irrigation is going on the abdomen should be gently rubbed. This stimulates peristaltic action of the entire intestinal tract, and favors elimination.

To unload the small intestine, drugs are necessary. Calomel is the best remedy, given in doses of from 1-10 to $1 / 2$ grain, repeated hourly until the typical spinach discharges are produced. In many cases this may be followed to advantage with castor-oil. In a majority of cases seen early, this treatment will arrest the disease.

When the disease is so arrested the next important point is in regard to the diet, because it should be remembered that these cases may easily suffer a relapse from renewed infection. It is often better to withhold milk for some days and use other varieties of food.

It is in this connection that certain proprietary articles are of service. Among these articles I would mention bovinine, Fairchild's panopepton, and liquid peptonoids. Mutton broth is an excellent article of diet, and may be the sole food for several days. It should be carefully prepared and all fat removed. In children over a year of age farinaceous infusion may be employed. The following, taken from $\mathrm{Jacobi}^{3}$, answers a good purpose: $5 \mathrm{oz}$. barley water, the white of one egg, from one to two teaspoonfuls of brandy or whisky, some salt and sugar. Small quantities of this may be given every few minutes.

When a milk diet is resumed, it should be thoroughly sterilized and properly diluted. In infants under a year of age, I prefer the milk and cream mixture. The formula which T usually employ is as follows:

3 Therapeutics of Infancy and Childhood, p. 225.

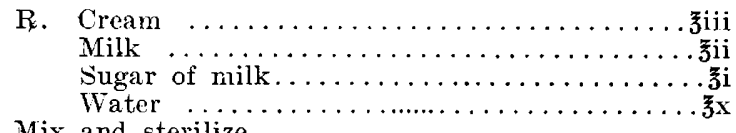

Mix and sterilize.

To each three or four ounces of this, when ready for feeding, one to two drams of lime water should be added. In preparing this the sugar should be dissolved in water and the cream and milk added.

This formula can easily be modified to suit individual cases. Cases that do not yield to the treatment already indicated, and this includes those that have persisted for a few days before medical advice is sought, require further attention.

Where the alvine discharges continue fetid, remedies directed to the disinfection of the alimentary tract are indicated. A remedy that has given me the best satisfaction is eudoxin. This may be given in doses of from $1 / 8$ to $1 / 2$ gr., in powder, combined with sugar of milk.

Where the discharges are acid in character, as indicated by the erythema about the buttocks, this remedy may be combined with an alkali, as subnitrate of bismuth or prepared chalk. In these cases also the bowels may be irrigated twice each day with a warm saline solution. Where there is much tympanites, it is of benefit to add to the water bicarbonate of soda one dram to the pint.

Where the diarrhea persists the question of the use of astringents suggests itself. My own belief is that it is better to use some remedy to check the action of the bowels, because frequent movements interfere with the rest and comfort of the patient. I have never been able to see any benefit from the vegetable astringents, even in those cases where they are retained by the stomach. of the preparations of opium I prefer either paregoric or Dover's powders. These allay irritation, arrest peristalsis, and give needed rest. A safe rule is to direct that the prescribed dose be given after each movement, thus as they become less frequent, less of the drug is given.

Where there is marked prostration, supportive measures are indicated. Alcoholic stimulants, externally and internally, are to be employed. Children bear stimulants well. Whisky is the better, as it is less likely to be adulterated than wines or brandies. For external use, equal parts of alcohol and hot water are better than alcohol alone. Where the heart is evidently weak, digitalis is an excellent remedy. I prefer the fluid extract, owing to the smallness of the dose, of which one drop may be given every two or three hours in water.

There are a certain number of cases that persist in spite of treatment. This class includes those eases where there is a true inflammatory lesion in the bowel, with a general sepsis. The discharges consist largely of mucus, epithelium, and blood. The treatment already indicated is appropriate to these cases, but often unavailing. Small doses of silver nitrate are sometimes of benefit. Sulphocarbolate of zinc is another remedy worthy of trial.

Whatever the reason may be, the fact remains that change of location is the most valuable treatment possible. I remember a case that I treated with indifferent success for five weeks and then suggested a change. The mother took the child to Spirit Lake, Iowa, and in one week, without any other general change in treatment, the child was restored to health. In another case seen by me in consultation a year ago, the disease had persisted for four weeks. The child was extremely emaciated and anorexia was complete. This patient was: sent to Wyoming, where it made a complete recovery. 
CHOLERA INFANTLI.

A few thoughts upon the subject of the treatment of the acute form of this disease. After irrigation of the bowel it is of benefit to use the following: $20 \mathrm{gr}$. of tannic acid dissolved in one pint of boiled water. This is injected into the bowel and retained by pressure against the buttocks. The acid probably coagulates any albuminoids that may remain in the bowel, and prevents their absorption. This treatment, which was suggested by Professor Vaughan, I have frequently employed, with decided benefit.

As the watery discharges rapidly produce collapse, it is good treatment to arrest the action of the bowels, after they have been irrigated. For this purpose I employ the method suggested by Holt, namely, the hypodermatic injection of morphin sulphate, gr. 1-100, with atropia sulphate, gr. 1-800. This will frequently arrest the action of the bowel for some hours, but should not be repeated within twelve hours.

Thirst is usually intense, but the stomach is so irritable that fluids are generally rejected. All kinds of foods should be strictly withheld. The most agreeuble drink is ice-cold sterilized water containing one drop of dilute nitric acid to each half ounce.

For collapse there is nothing equal to the subcutaneous infusion of normal salt solution. The only apparatus necessary is a hypodermic needle and fountain syringe. This supplies the system with water in a manner so that it can not be rejected. When the extremities are cold the hot sheet pack is indicated. Mustard may be added to the water in which the sheet is dipped, with advantage.

Nervous symptoms are probably due to either fever, the absorption of toxins, or both, and when prominent, generally indicate a fatal termination. The ice-cap to the head can safely be employed. A solution of bromid of potassium and chloral hydrate may be given per rectum.

Coal tar drugs are seldom to be employed.

1312 N. 40th Street.

\section{SURGICAL TREATMENT OF ACUTE PERITONITIS.* \\ BY A. F. HOUSE, M.D. \\ Surgeon to St. Clair Hospital; Consulting Surgeon to the German Hospital. CLEVELAND, OHIO.}

In presenting my paper on the surgical treatment of acute peritonitis I have not thought it necessary to review my experimental work, or to report all my cases operated upon. Neither is it possible in a short paper to enter into a discussion of the anatomy, etiology, pathology and the various clinical phases found in this formidable disease, because inflammation of this membrane may have so many different causes and assume such varied clinical aspects that it is difficult to formulate a uniform and satisfactory classification. Suffice it to say that, anatomically and physiologically considered, the peritoneal cavity may be said to be a large lymph-sac, and noted for its capacity of absorption. This capacity is not surprising when we take into consideration that in its parietal and visceral enfoldings, it presents nearly as large a surface as the entire integumentary covering of the body.

Idiopathic peritonitis is considered doubtful by most modern pathologists, and it has become an established

*Presented to the Section on Surgery and Anatomy, at the Fiftieth Annual Meeting of the American Medical Association, held at Columbus, Ohio, June 6-9, 1899. practice to search for a local cause in all cases of peritonitis. I believe in every instance, micro-organisms from some source or other have gained access to the peritoneal cavity. Wounds of the peritoneum heal with remarkable rapidity if the surfaces are kept in uninterrupted contact; in a few hours, adhesion takes place between the united parts by means of plastic material and is termed "plastic peritonitis." This is purely a regenerative process and should not be classed with the inflammatory affections.

Of the various structures of the body, the peritoneum, without question, is one of the most susceptible to septic influences, and it has become an established fact that different portions of the peritoneum present different degrees of vulnerability to sepsis. The most sensitive region is that over the small intestines. The parietal peritoneum is much less susceptible to infection. When peritonitis is developed away from the small intestinal area, it is apt to be localized. This is shown by the course of peritonitis in the iliac fossa, in the subphrenic region and in the pelvis.

Septic and suppurative peritonitis are, etiologically, identical; however, clinically they differ in that acute septic peritonitis is generally diffuse and leads to a rapidly fatal termination, while both forms are caused by a pus organism. Suppurative peritonitis is more generally local or circumscribed and more often amenable to surgical treatment. It is quite likely that the most common form of peritonitis is that caused by infection from some portion of the alimentary canal, as in appendicitis or acute obstructions and the various forms of ulceration of the bowels. The organism generally found in these cases is the bacillus coli communis. Still there are certain other infections which pursue quite as fulminating a course as those from the alimentary tract, such as for instance results from faulty technic, in which a virulent germ is introduced by instruments, sponges or fingers. We also know that the reproductive organs are often the cause of a virulent peritonitis, as in certain forms of metritis or salpingitis. It may be well also to menticn here certain forms of inflammation of the liver and gall-bladder and the rupture of abscesses of various parts of the body as producing peritonitis. 'The microorganism usually found in these cases is the streptococcus. In rare instances, perhaps, some of the milder pus cocci seem to have an unusual virulence. I believe, barring faulty asepsis, that peritonitis caused by infection from the intestine is the most rapid in its progress and the most fatal in its results. Whether the colon bacillus in its growth is an effect rather than a cause, I am unable to say. Its constant presence in all cases of peritonitis of intestinal origin justifies the opinion that it has at least a great influence as a causative factor. The symptoms of acute peritonitis when operative interference is advisable and not without hope may be grouped as follows:

1. Pain of varying degree; either local and becoming general, or general becoming local, according to cause. This pain is very frequently referred to the region of the umbilicus, even when the seat of infection is remote from that point.

2. Tenderness, general becoming local, or local becoming general, as a result of pain. Marked rigidity of the abdominal muscles is present, and in many instances this rigidity is more marked over the portion of the peritoneal cavity in which the infection commenced. The importance of early rigidity is a symptom of the utmost value, and my experience leads me to fear that its aid in 